Modelling, Analysis and Simulation

Modelling, Analysis and Simulation
MAS Forcing relations for homoclinic orbits of the Smale
horseshoe map

P.J. Collins

RePOrt MAS-E04 19 NOVember 2004 
CWI is the National Research Institute for Mathematics and Computer Science. It is sponsored by the Netherlands Organization for Scientific Research (NWO).

CWI is a founding member of ERCIM, the European Research Consortium for Informatics and Mathematics.

CWI's research has a theme-oriented structure and is grouped into four clusters. Listed below are the names of the clusters and in parentheses their acronyms.

Probability, Networks and Algorithms (PNA)

Software Engineering (SEN)

Modelling, Analysis and Simulation (MAS)

Information Systems (INS)

Copyright (C) 2004, Stichting Centrum voor Wiskunde en Informatica

P.O. Box 94079, 1090 GB Amsterdam (NL)

Kruislaan 413, 1098 SJ Amsterdam (NL)

Telephone +31205929333

Telefax +31205924199

ISSN 1386-3703 


\title{
Forcing relations for homoclinic orbits of the Smale horseshoe map
}

\begin{abstract}
An important problem in the dynamics of surface homeomorphisms is determining the forcing relation between orbits. The forcing relation between periodic orbits can be computed using existing algorithms. Here we consider forcing relations between homoclinic orbits. We outline a general procedure for computing the forcing relation, and apply this to compute the equivalence and forcing relations for homoclinic orbits of the Smale horseshoe map.
\end{abstract}

2000 Mathematics Subject Classification: 37E30; 37C27; 37B10; 37E25.

Keywords and Phrases: Homoclinic orbit; Forcing relation; Smale horseshoe; Braid type.

Note: This work was partially funded by Leverhulme Special Research Fellowship SRF/4/9900172. 



\title{
Forcing relations for homoclinic orbits of the Smale horseshoe map
}

\author{
Pieter Collins * \\ Centrum voor Wiskunde en Informatica, P.O. Box 94079, \\ 1090 GB Amsterdam, The Netherlands \\ Email: Pieter.Collins@cwi.nl
}

12 November 2004

\begin{abstract}
An important problem in the dynamics of surface homeomorphisms is determining the forcing relation between orbits. The forcing relation between periodic orbits can be computed using existing algorithms. Here we consider forcing relations between homoclinic orbits. We outline a general procedure for computing the forcing relation, and apply this to compute the equivalence and forcing relations for homoclinic orbits of the Smale horseshoe map.
\end{abstract}

Keywords: Homoclinic orbit; Forcing relation; Smale horseshoe; Braid type.

AMS Subject Classifications: 37E30; 37C27; 37B10; 37E25.

\section{Introduction}

In this paper, we study the braid equivalence and forcing relation for homoclinic orbits of the Smale horseshoe map. This problem is important in obtaining a detailed understanding of the chaotic behaviour of general surface diffeomorphisms. The braid type of an orbit gives a combinatorial way of specifying the orbit, and the forcing relation places restrictions of the coexistence of orbits of different braid types. For systems exhibiting full-blown chaotic behaviour, we can then deduce the existence of infinitely many orbits from knowledge of just a single orbit, and also obtain a lower bound on the topological entropy. For parameterised families of systems exhibiting a transition to chaos, the forcing relation gives information on the order in which different orbits are created. The forcing relation for homoclinic orbits can also be used to give information about the periodic orbits of the system. We restrict to the Smale horseshoe map since this provides a model of chaotic dynamics in two dimensions which is universal (in a combinatorial sense), for which a natural symbolic description of orbits is available, and which models other important systems, such as the Hénon map.

Chaotic behaviour and the forcing relation is well-understood for one-dimensional (non-invertible) maps. Sharkovskii's theorem [23] describes the forcing relation of periodic orbits of interval maps in terms of the period. For unimodal interval maps, the kneading theory of Milnor and Thurston [21] shows that periodic orbits are created in a linear ordering. The kneading theory has been partially generalised to non-unimodal maps [1] to give a fairly complete picture of the bifurcation structure.

For systems in two-dimensions, the situation is more complicated and still not well-understood. It is still possible to obtain considerable information from a single orbit, but periodic orbits are only partially ordered [3] by the forcing relation. This makes the study of surface diffeomorphisms particularly

${ }^{*}$ This work was partially funded by Leverhulme Special Research Fellowship SRF/4/9900172. The author would like to thank Toby Hall and Eiko Kin for numerous comments and suggestions which were invaluable in the writing of this paper. 
interesting; the dynamics is much richer than that of one-dimensional maps, but there is enough structure that a fairly complete understanding of chaotic dynamics is feasible.

Considerable attention in the literature has been paid to the Hénon family, a two-dimensional analogue of the one-dimensional quadratic family, and to the Smale horseshoe map, an analogue of the tent map, and a theoretically tractable chaotic system. It has been shown that the Hénon map has a full Smale horseshoe for certain parameter values, and a partially-formed horseshoe for others [12]. In fact, any $C^{1+\epsilon}$ surface diffeomorphism with positive topological entropy must have a horseshoe [17], so the Smale horseshoe map is pivotal in studying chaos in two-dimensions. Further, the third iterate of the Smale horseshoe map contains periodic orbits of all braid types [18], so is "combinatorially universal".

The forcing relation for periodic orbits of surface diffeomorphisms can be studied using the NielsenThurston theory of surface homeomorphisms [24]. This gives a classification of surface homeomorphisms up to isotopy, and provides a canonical diffeomorphism in each isotopy class which minimises the topological entropy and has minimal orbit structure (see [14, 4]). A number of algorithms exist to compute the forcing relation $[2,13,19]$.

A conjecture of Hall and de Carvalho [10] suggests that the periodic orbits of the Smale horseshoe map are partitioned into families on which the forcing relation restricts to a linear order, and that each family has an associated homoclinic orbit which forces all periodic orbits of the family. The forcing relation between families can be determined from a knowledge of the forcing relation between the associated homoclinic orbits. If the conjecture is true, being able to compute the forcing relation between homoclinic orbits is an important problem in determining the complete forcing relation. Partial results on the conjecture are given in [11].

In this paper we compute the braid equivalence and forcing relations for homoclinic orbits of the Smale horseshoe map using the trellis theory developed in $[7,8]$. The braid type of a homoclinic orbit to a saddle fixed point can be described by the trellis formed by finite pieces of the stable and unstable manifolds of the saddle fixed point. The trellis can be represented combinatorially by giving the relative ordering of the intersection points along the stable and unstable manifolds. Since approximations to trellises can be computed numerically for a given diffeomorphism, we can, in theory, extract the combinatorics of a numerically computed trellis to find the braid type. The results of this paper, however, use a purely combinatorial construction of the trellis from the symbolic coding of the homoclinic orbit.

The fundamental operation on trellises is that of pruning, related to the pruning isotopies of de Carvalho [9]. However, the pruning theory for trellises is much simpler than the general theory, and has a straightforward formulation in terms of the combinatorics of the trellis. We compute the trellis associated with a given homoclinic horseshoe orbit by pruning a trellis for the Smale horseshoe map relative to the given orbit. The braid equivalence relation is given by comparing the combinatorics of the resulting trellis, and the forcing relation can be given by following the other orbits through the pruning. By computing the graph representative of the trellis, a lower bound for the topological entropy forced by the homoclinic orbit can be found.

A number of other authors have considered forcing relations for homoclinic orbits and tangles. RomKedar [22] gave forcing relations and entropy bounds for some infinite families of trellises. McRobie and Thompson [20] discussed the possible bifurcation sequences associated with the break-up of horseshoes in a driven oscillator using methods similar to our prunings. Methods for the computation of the forcing relation for homoclinic orbits have been given by Handel [15] and Hulme [16]. The methods outlined in this paper have the advantage of being applicable to general trellises and homoclinic/heteroclinic orbits, and the computations are straightforward to implement. Further, this is the first paper to present numerical computations of forcing relations between homoclinic orbits.

The paper is organised as follows. In Section 2, we describe the combinatorics of periodic and homoclinic orbits of the Smale horseshoe map, and introduce the braid type of a homoclinic orbit. In Section 3 we introduce the results of trellis theory which are necessary for the calculations. In Section 4 we describe the algorithms used to compute the braid equivalence and forcing relations, and discuss the implementation of the pruning procedure. In Section 5 we present the results of numerical computations of the equivalence and forcing relations for homoclinic orbits of the Smale horseshoe map with short symbolic codings. Finally, we give some conclusions and open problems in Section 6.

The computations of this paper were performed using the $\mathrm{C}++$ library "trellis", which is freely available for download [6]. This package allows computations on combinatorial trellises, including the computation of the trellis associated with a horseshoe homoclinic orbit, and the computation of the graph representative of the trellis. 


\section{Horseshoe Orbits}

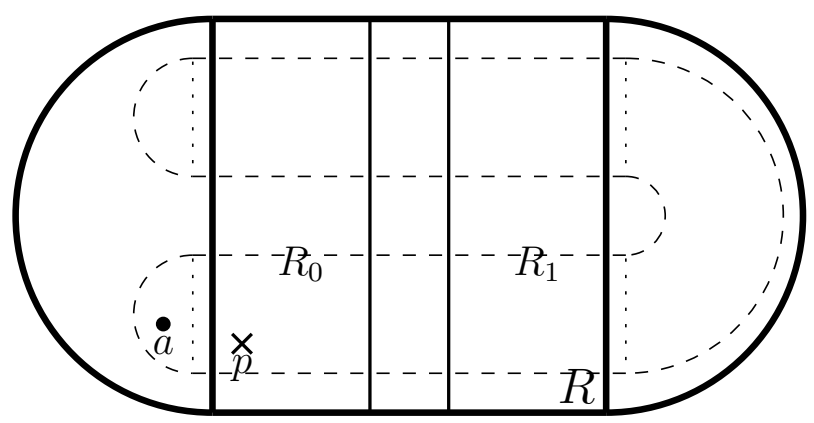

Figure 1: The Smale horseshoe map

In this paper, the model of the Smale horseshoe map $f: S^{2} \rightarrow S^{2}$ depicted in Figure 1 will be used. The stadium-shaped domain shown, consisting of two half-discs and a square $R$, is mapped into itself as in an orientation-preserving way as indicated by the dotted lines, with a stable fixed point $a$ in the left hemisphere. The map is then extended to a homeomorphism of $S^{2}$ with a repelling fixed point at $\infty$ whose basin includes the complement of the stadium domain. The saddle fixed point of negative index (i.e. positive eigenvalues) is denoted $p$.

The non-wandering set $\Omega(f)$ consists of the fixed points $a$ and $\infty$, together with a Cantor set

$$
\Lambda=\left\{x \in S^{2}: f^{n}(x) \in R \text { for all } n \in \mathbb{Z}\right\} .
$$

Since $\Lambda$ is contained in the union of the rectangles $R_{0}$ and $R_{1}$, symbolic dynamics can be introduced in the usual way, providing an itinerary homeomorphism

$$
k: \Lambda \rightarrow \Sigma_{2}=\{0,1\}^{\mathbb{Z}},
$$

with the property that $\sigma(k(x))=k(f(x))$ for all $x \in \Lambda$ (where $\sigma: \Sigma_{2} \rightarrow \Sigma_{2}$ is the shift map). The code of an orbit $H$ in $\Lambda$ is the bi-infinite sequence given by the itinerary of any of its points.

The itinerary of a point $x$ is periodic of period $n$ if and only if $x$ is a period $n$ point of $F$. This paper is concerned with orbits which are homoclinic to $p$, and the term homoclinic orbit will be used exclusively to mean such orbits. A point $x \in \Lambda$ is homoclinic if and only if its itinerary consists of finitely many 1 's.

Definition 2.1 (Core) Let $H$ be a homoclinic orbit of the horseshoe. The core of $H$ is the longest word in the code of $H$ which begins and ends with 1.

The signature of $H$ is equal to the length of the core minus one. Thus, for example, the point with itinerary $\overline{0} 110 \cdot 0101 \overline{0}$ is a point of the homoclinic orbit $\overline{0} 1100101 \overline{0}$ with core 1100101 and signature 6 . The primary homoclinic orbits are those with cores 1 and 11; these two orbits have the same homoclinic braid type, are forced by every other homoclinic orbit, but do not force any other periodic or homoclinic orbit. By contrast, the orbits with cores 111 and 101 will be shown to force all periodic and homoclinic orbits of the horseshoe (cf. [15]).

Definition 2.2 (Decoration) The decoration of $H$ is defined to be $*$ if $H$ has code $\overline{0} 1_{1}^{0} 1 \overline{0}$, · if $H$ has code $\overline{0} 1_{11}^{00} 1 \overline{0}$ and $c$ if $H$ has code $\overline{0} 1_{1}^{0} c_{1}^{0} 1 \overline{0}$.

Orbits of the Smale horseshoe map with the same decoration have the same braid type, as defined below.

Definition 2.3 (Braid type) The braid type of a homoclinic orbit $H$ of a surface diffeomorphism $f$, denoted $\mathrm{BT}[H ; f]$ is the conjugacy class of the isotopy class of $f$ relative to $H$.

In other words, orbits $H$ of $f$ and $H^{\prime}$ of $f^{\prime}$ have the same braid type if there is an isotopy $\left(f_{t}\right)$ and a diffeomorphism $h$ such that $f=f_{0}, H$ is a homoclinic orbit of $f_{t}$ for all $t, H^{\prime}=h(H)$ and $f^{\prime}=h \circ f_{1} \circ h^{-1}$.

Definition 2.4 (Forcing) A braid type BT forces $\mathrm{BT}^{\prime}$ if every homeomorphism $f$ with a homoclinic orbit $H$ with $\mathrm{BT}(H, f)=\mathrm{BT}$ has a homoclinic orbit $H^{\prime}$ with $\mathrm{BT}\left(H^{\prime}, f\right)=\mathrm{BT}^{\prime}$. 


\section{Horseshoe trellises}

In this section those aspects of trellis theory which will be used later are reviewed. Trellis theory is applicable in a much more general setting (see [8] for full details), but here the key definitions and results are presented in a manner tailored for the study of horseshoe trellises. All results stated in this section can be found in [8].

The key ideas presented are as follows. A trellis is a finite portion of the tangle of stable and unstable manifolds of a saddle fixed point. Starting with the familiar tangle of the full horseshoe, the full horseshoe trellis of signature $n$ can be defined for each integer $n \geq 2$ : it has longer and longer stable and unstable branches as $n$ increases. Given a horseshoe homoclinic orbit, the full horseshoe trellis of appropriate signature can be pruned, by removing as many intersections as possible without disturbing the given homoclinic orbit. This pruned trellis is a complete invariant of homoclinic braid type, and so the technique can be used to determine whether or not two given homoclinic orbits have the same braid type.

Given a trellis (and the action of a diffeomorphism on it), there is a lower bound on the dynamics of any diffeomorphism which has such a trellis. This minimal dynamics can be computed as the dynamics of a tree map, using techniques similar to those developed for computing train tracks for periodic orbits by Bestvina and Handel [2] and Franks and Misiurewicz [13].

The dynamics forced by a given horseshoe homoclinic orbit can thus be determined by finding the appropriate pruned horseshoe trellis, and calculating the associated tree map. In this paper, we are only concerned with forcing relations between homoclinic orbits.

\subsection{The full horseshoe trellis}

Definition 3.1 (Trellis) Let $f: S^{2} \rightarrow S^{2}$ be a diffeomorphism, and $p$ be a hyperbolic saddle fixed point of $f$. Then a trellis for $f($ at $p)$ is a pair $T=\left(T^{U}, T^{S}\right)$, where $T^{U}$ and $T^{S}$ are intervals in $W^{U}(f ; p)$ and $W^{S}(f ; p)$ respectively containing $p$. (Here, $W^{U}(f ; p)$ and $W^{S}(f ; p)$ denote the unstable and stable manifolds, respectively, of $f$ at $p$.) Given a trellis $T=\left(T^{U}, T^{S}\right)$, denote by $T^{V}$ the set of intersections of $T^{U}$ and $T^{S}$. The trellis is transverse if all of its intersection points are transverse.

Since all trellises considered in this paper will be transverse, and hence the word trellis will be understood to mean transverse trellis.

Definition 3.2 (Segment) Let $T=\left(T^{U}, T^{S}\right)$ be a trellis. A segment of $T$ is a closed subinterval of either $T^{U}$ or $T^{S}$ with endpoints in $T^{V}$ but interior disjoint from $T^{V}$. The segment is called unstable or stable according as it is a subinterval of $T^{U}$ or of $T^{S}$.

Definition 3.3 (Region, bigon) Let $T=\left(T^{U}, T^{S}\right)$ be a trellis. Then a region of $T$ is the closure of a component of $S^{2} \backslash\left(T^{U} \cup T^{S}\right)$. A bigon of $T$ is a region bounded by two segments (one unstable and one stable).

Definition 3.4 (Full horseshoe trellis) Let $f$ be the Smale horseshoe map, and $p$ be the fixed point with code $\overline{0}$. Let $q_{0}$ be the homoclinic point with itinerary $\overline{0} \cdot 1 \overline{0}$, and $q_{k}=f^{k}\left(q_{0}\right)$. Given $i \geq 0$ and $j \leq 0$, denote by $T_{i}^{U}$ an interval in $W^{U}(f ; p)$ with end intersections $p$ and $q_{i}$, and by $T_{j}^{S}$ the interval in $W^{S}(f ; p)$ with end points $p$ and $q_{j}$. For $n \geq 2$, a full horseshoe trellis of signature $n$ is a trellis $T=\left(T_{i}^{U}, T_{j}^{S}\right)$ such that $i-j=n$.

It is clear that all full horseshoe trellises as defined above are differentiably conjugate.

Example 3.5 The full horseshoe trellis of signature 2 is depicted in Figure 2(a). The chaotic dynamics is supported in the regions labelled $R_{0}$ and $R_{1}$. All points in $R_{U}$ are in the basin of the attracting fixed point $a$, and all points in the interior of $R_{U}$ are in the basin of the repelling point at infinity. The point $r_{0}$ has itinerary $\overline{0} 1 \cdot 01 \overline{0}$, and the point $r_{1}$ has itinerary $\overline{0} 1 \cdot 11 \overline{0}$. The full horseshoe trellis of signatures 3 is depicted in Figure 2(b). 


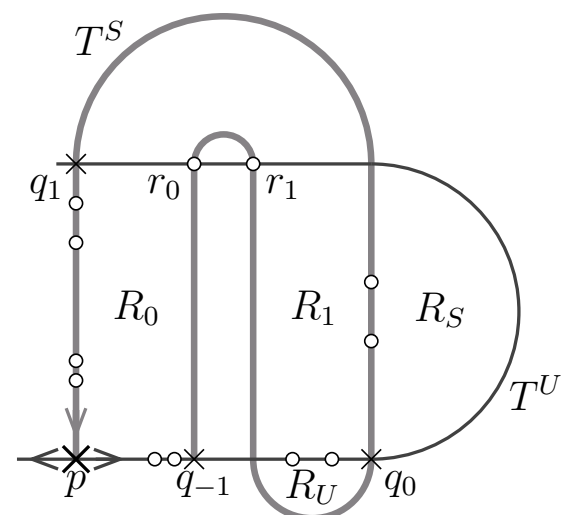

(a)

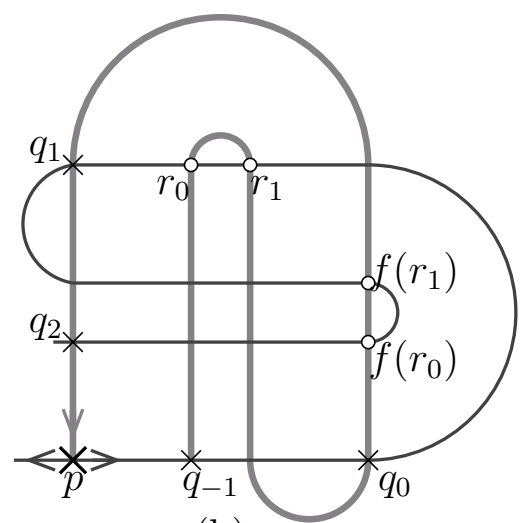

(b)

Figure 2: (a) The full horseshoe trellis with signature 2. (b) The full horseshoe trellis with signature 3 .

\subsection{Pruning isotopies}

Given a trellis $T$ for a diffeomorphism $f$, a pruning isotopy is an isotopy which removes the intersections on the boundary of one or more bigons of $f$. To be more precise, it is an isotopy from $f$ to a diffeomorphism $f^{\prime}$ which has a trellis $T^{\prime}$ obtained from $T$ by removing such intersections. There are two possibilities; we can either remove both intersections of a single bigon, as depicted in Figure 3(a), or remove intersections from two neighbouring bigons, changing the orientation of the crossing at the remaining intersection, as depicted in Figure 3(b).

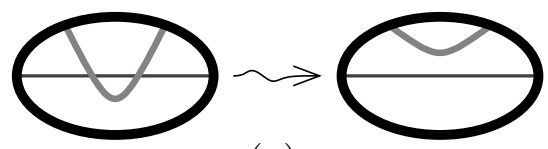

(a)

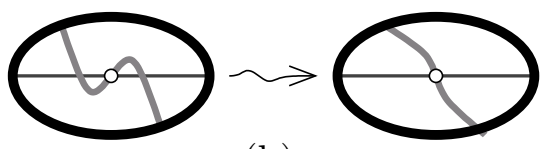

(b)

Figure 3: The local effect of pruning isotopies on a trellis

However, an isotopy of the diffeomorphism $f$ supported in some open set $U$ will also change the trellis outside of $U$. If we are trying to reduce the number of intersections of $T$, we need to ensure that no other intersections are created when we remove intersections locally. This gives rise to the notion of an inner bigon

Definition 3.6 (Inner bigon) A bigon $B$ is inner if $B \cap \bigcup_{n \in \mathbb{Z}} f^{n}\left(T^{V}\right)=B \cap T^{V}$. i.e. A bigon is $B$ inner if the only intersections of $B$ with the orbits of the intersection points of the trellis are the vertices of $B$.

The following result follows from the proof of Theorem 3.5 of [8].

Theorem 3.7 (Pruning away an inner bigon) Let $T$ be a trellis of a diffeomorphism $f$. Suppose either that $B$ is an inner bigon with vertices $v_{0}$ and $v_{1}$, or that $B_{0}$ and $B_{1}$ are inner bigons with a common vertex $v$ and other vertices $v_{0}$ and $v_{1}$ on different orbits. Then there is a diffeomorphism $h: S^{2} \rightarrow S^{2}$, which we can take to be supported on a neighbourhood $U$ of $B$ or $B_{0} \cup B_{1}$, such that $f^{\prime}=f \circ h$ has a trellis $T^{\prime}$ with the same intersections apart from those on the orbits of $v_{0}$ and $v_{1}$ under $f$.

The trellis $T^{\prime}$ is obtained by removing all the intersections of $T$ contained in the orbit of $U$. The diffeomorphism $f^{\prime}$ is isotopic to $f$, and a general pruning isotopy can be constructed as a sequence of isotopies pruning away an inner bigon. 
Example 3.8 Figure 4a) depicts the full horseshoe trellis of signature 3, and a shaded neighbourhood $U$ of an inner bigon $B$, together with its image. Pruning away the bigon $B$ yields a diffeomorphism $f^{\prime}$ with trellis $T^{\prime}$ as shown in b). Pruning away the bigon $B^{\prime}$ yields a diffeomorphism $f^{\prime \prime}$ with the trellis $T^{\prime \prime}$ depicted in c).

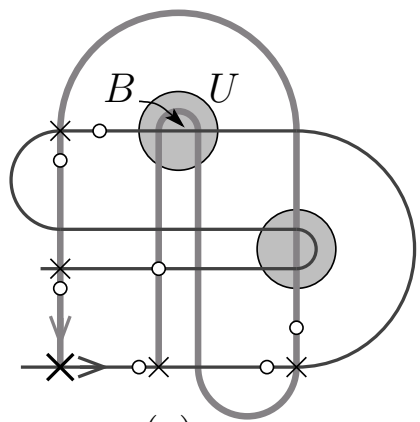

(a)

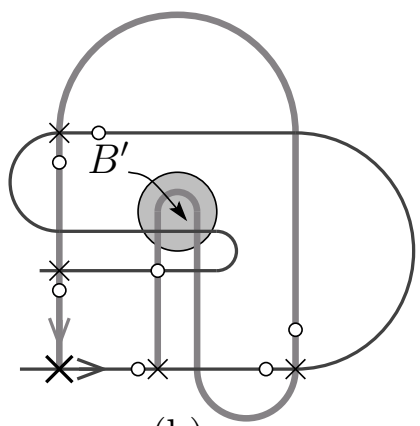

(b)

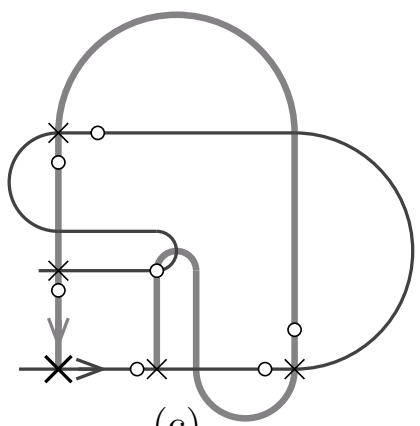

(c)

Figure 4: Pruning away inner bigons in the horseshoe trellis

\subsection{Horseshoe trellises}

A trellis obtained by pruning the full horseshoe trellis as in Example 3.8 is called a horseshoe trellis:

Definition 3.9 (Horseshoe trellis) A horseshoe trellis is a trellis $T$ obtained from the full horseshoe trellis by a pruning isotopy (i.e. by pruning away a sequence of inner bigons).

A horseshoe trellis can be associated to each homoclinic orbit of the horseshoe, by pruning away as many inner bigons as possible without touching the homoclinic orbit. It is trivial that the signature of a horseshoe homoclinic orbit $H$ is equal to the least integer $n$ such that $H$ is an intersection of the full horseshoe trellis of signature $n$.

Definition 3.10 (Trellis forced by a homoclinic orbit) Let $H$ be a horseshoe homoclinic orbit of signature $n$, and let $m \geq n$. The trellis of signature $m$ forced by $H$ is the trellis $T$ obtained from the full horseshoe trellis of signature $m$ by pruning away inner bigons as much as possible without removing points of $H$.

Example 3.11 The white circles in Figure 4 represent points of the homoclinic orbit $H$ with code $\overline{0} 1001 \overline{0}$ (which thus has signature 3). The trellis of Figure 4c) is thus the trellis forced by this homoclinic orbit. Note that every bigon has a point of $H$ on its boundary.

This method makes it possible to determine whether or not two horseshoe homoclinic orbits have the same homoclinic braid type:

Definition 3.12 (Trellis type) Let $T$ and $T^{\prime}$ be horseshoe trellises for diffeomorphism $f$ and $f^{\prime}$ respectively. We say that $(f ; T)$ and $\left(f^{\prime} ; T^{\prime}\right)$ have the same trellis type if there is a diffeomorphism $g$ isotopic to $f$ relative to $T$, and a homeomorphism $h: S^{2} \rightarrow S^{2}$ such that $h(T)=T^{\prime}$ and $h^{-1} \circ f^{\prime} \circ h=g$.

We denote the trellis type containing $(f ; T)$ by $[f ; T]$.

For horseshoe trellises, the trellis type is determined by the geometry of the trellis:

Theorem 3.13 Let $T$ and $T^{\prime}$ be horseshoe trellises for diffeomorphism $f$ and $f^{\prime}$ respectively. Then $(f ; T)$ and $\left.f^{\prime} ; T^{\prime}\right)$ have the same trellis type if and only if $T$ and $T^{\prime}$ are diffeomorphic.

Proof: It suffices to consider the case $T=T^{\prime}$. Since the points with itinerary $\overline{0} 1 \overline{0}$ all lie on a single homoclinic orbit, we can deduce the action of $f$ and $f^{\prime}$ on all the vertices of $T$ from the action of this orbit simply by counting vertices in each fundamental domain. The result follows since all regions of $T$ are simply-connected, so the isotopy class is determined by the action on the segments. 
Since a horseshoe trellis type is fully determined by the geometry of the trellis, we define the type of a horseshoe trellis $T$ to be the type of $(f ; T)$ for any diffeomorphism $f$ with trellis $T$ which can be obtained by pruning away inner bigons.

Horseshoe trellises $(f ; T)$ and $\left(f^{\prime} ; T^{\prime}\right)$ have the same type if and only if the trellises $T$ and $T^{\prime}$ are homeomorphic, and this occurs if and only if the orderings of the intersections on the stable and unstable manifolds are the same.

Definition 3.14 (Intersection ordering) Let $(f ; T)$ be a horseshoe trellis, with intersections $T^{V}=$ $\left\{v_{i}: i=0 \ldots n-1\right\}$ such that $v_{i}<_{u} v_{i+1}$ (i.e. $v_{i}$ is closer to $p$ along the unstable manifold). Then the relative ordering of the unstable and stable manifolds is the permutation $\pi_{T}$ such that $v_{\pi_{T}(i)}<_{s} v_{\pi_{T}(j)}$ if and only if $\pi_{T}(i)<\pi_{T}(j)$.

The following result gives a computable criterion for the equivalence of horseshoe trellises.

Theorem 3.15 Let $T$ and $T^{\prime}$ be horseshoe trellises. Then $T$ and $T^{\prime}$ have the same trellis type if and only if $\pi_{T}=\pi_{T^{\prime}}$.

The following result is immediate from Theorem 2 of [8], and shows that a homoclinic braid type is determined by the geometry of the trellis obtained by pruning up to the given orbit. In particular, homoclinic orbits can only have the same braid type if they have the same signature (i.e. their cores have the same length).

Theorem 3.16 Let $H$ and $H^{\prime}$ be horseshoe homoclinic orbits of signatures $n$ and $n^{\prime}$, and let $T$ and $T^{\prime}$ be the trellises of signature $m \geq \max \left\{n, n^{\prime}\right\}$ forced by them. Then $H$ and $H^{\prime}$ have the same homoclinic braid type if and only if $T$ and $T^{\prime}$ have the same trellis type.

The following result follows from the main theorem (Theorem 1) of [5] which shows that the dynamics forced by a trellis is minimal in the isotopy class.

Theorem 3.17 Let $H$ and $H^{\prime}$ be horseshoe homoclinic orbits of signatures $n$ and $n^{\prime}$, and let $T$ and $T^{\prime}$ be the trellises of signature $m \geq \max \left\{n, n^{\prime}\right\}$ forced by $H$. The homoclinic braid type of $H$ forces the braid type of $H^{\prime}$ if and only if $T$ has an intersection on an orbit with the same braid type as $H^{\prime}$.

\section{Computation of the equivalence and forcing relations}

We now give a brief outline of the algorithm used to compute the equivalence and forcing relations for horseshoe homoclinic orbits, and then discuss the implementation details of the pruning procedure.

\subsection{Algorithm}

To compute the trellis forced by the orbit $H$, a full horseshoe trellis of the same signature as $H$ is constructed, and then pruned relative to the orbit $H$ and the orbit with code $\overline{0} 1 \overline{0}$, the latter being used to fix the end intersections. The pruning process sweeps through the trellis, pruning whenever an inner bigon is found whose vertices are not points of $H$, or a pair of inner bigons is found whose outer vertices are not points of $H$.

The equivalence relation for orbits of signature $n$ is determined by first computing all the trellises of signature $n$ associated with the homoclinic orbits. The homoclinic orbits of the same decoration are known to have the same braid type, so only one orbit with each decoration is needed. Since the trellis is a braid type invariant by Theorem 3.16, and the relative intersection ordering characterises horseshoe orbits by Theorem 3.15, the relative intersection ordering is computed for each orbit, and the orbits partitioned into equivalence classes accordingly. To compute the equivalence relation between homoclinic horseshoe orbits, it suffices to compute the trellis of signature $n$ forced by each.

Since the (horseshoe) itineraries of intersections can be continued through the pruning, the codes of the remaining orbits can be recovered from the pruned trellis. Note, though, that in order to show that the homoclinic braid type of a homoclinic orbit $H$ does not force that of a homoclinic orbit $H^{\prime}$, it is necessary to show that none of the homoclinic orbits of the same type as $H^{\prime}$ persist through the pruning. 
Thus it is necessary to compute equivalences in order to be able to compute the forcing relation. Hence, to compute whether $\mathrm{BT}(H)$ forces $\mathrm{BT}\left(H^{\prime}\right)$ where $H$ has signature $n$ and $H^{\prime}$ has signature $n^{\prime}$, we need to compute all trellises of signature $n^{\prime}$ to determine the equivalence relation on braid types of signature $n^{\prime}$. We then compute the trellis of signature $\max \left\{n, n^{\prime}\right\}$ forced by $H$, and determine if it contains an orbit of braid type $\mathrm{BT}\left(n^{\prime}\right)$.

The topological entropy forced by the homoclinic orbit of a given braid type was computed by finding the graph representative, as detailed in $[7,8]$.

\subsection{Implementation of pruning}

The most natural implementation of a trellis type is as a list of vertices with image information. Hence an intersection can be represented as

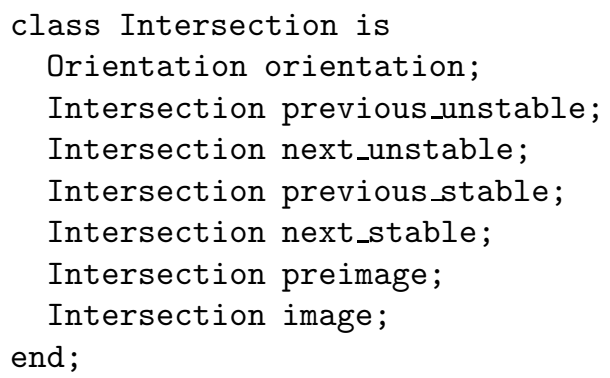

Here, each Intersection data element is a reference to another intersection, which may be void, and the orientation is either positive or negative, depending on the orientation of the intersections of the periodic orbit. We remark that this contains more information than is needed to specify the trellis structure; it suffices to store the next_unstable, next_stable image intersections. However, at least one of previous_unstable or previous_stable is required for the algorithm, and preimage is useful for general trellises. Note that the orientations for horseshoe trellises always alternate between positive and negative.

The main procedure needs to detect and prune inner bigons, as shown in Figure 3(a). Detecting bigons and inner bigons can be accomplished by the predicates is_bigon and is_inner_bigon. We assume throughout that the function arguments are not void.

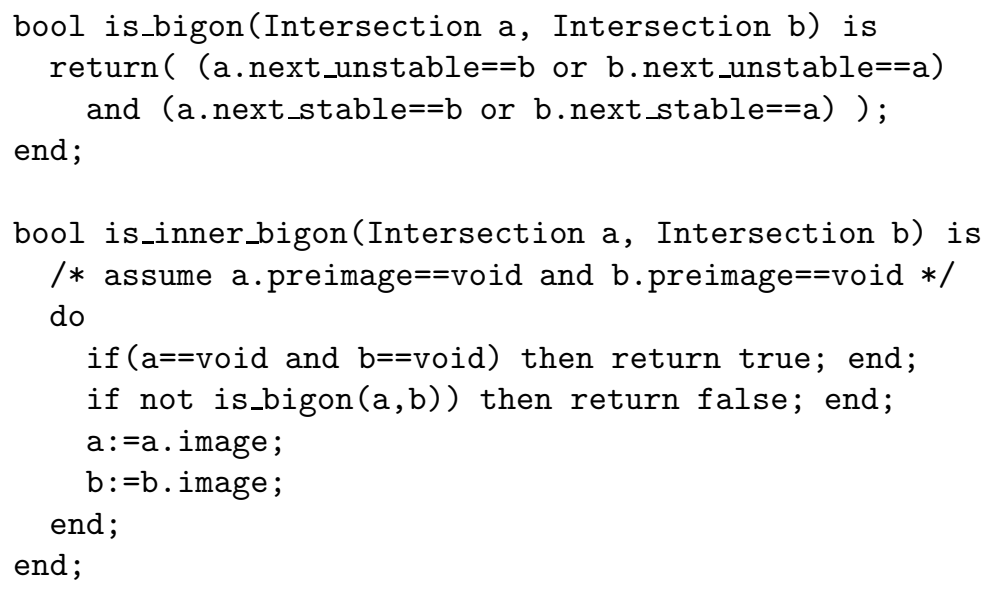

The pruning is performed by the procedures prune_bigon and prune_inner_bigon described below. The vertices $\mathrm{a}$ and $\mathrm{b}$ are deleted following a prune_bigon function.

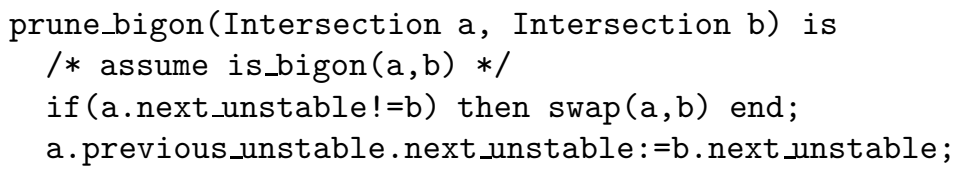




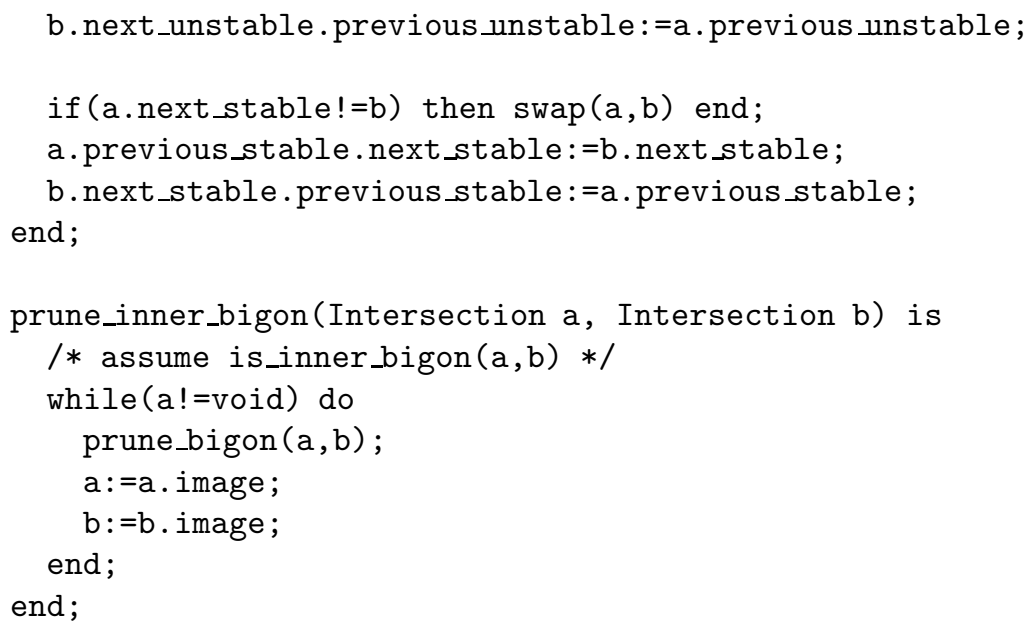

The procedure prune_inner_bigon can be easily constructed from prune_bigon. Detecting and removing the intersections of two adjacent bigons as shown in Figure 3(b) can be carried out as follows: The vertices a and c may be deleted after a prune_bigon_pair procedure.

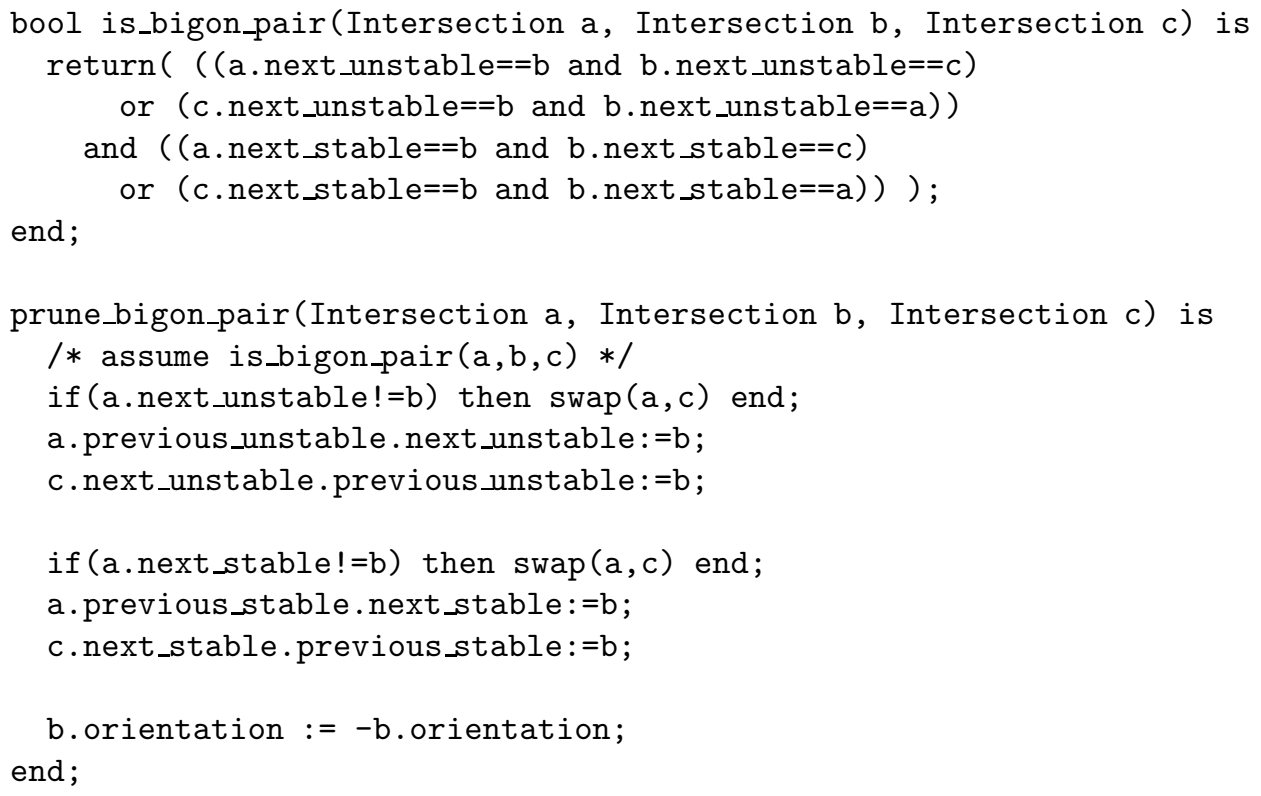

When dealing with trellises which are not horseshoe trellises, extra code is needed to take care of degenerate cases involving endpoints. In particular, in the function is_inner_bigon, an extra line

$$
\text { if ( } a==\text { void or } b==\text { void) then return false; end; }
$$

is needed in case an endpoint occurs between a and b. These procedures can be further optimised; we present them in the form given here to show explicitly the steps required, omitting only the details of garbage collection.

\section{$5 \quad$ Numerical results}

In this section we compute, for horseshoe homoclinic orbits with short cores, the equivalence classes under the relation of having the same homoclinic braid type, and the forcing relation. 


\begin{tabular}{|c|c|l|}
\hline Sig & Dec & Ordering \\
\hline 2 & $*$ & $\mathbf{0}, \boldsymbol{7}, 4,3,2, \mathbf{5}, \mathbf{6}, 1$ \\
\hline 3 & $\cdot$ & $\mathbf{0}, 9,6,5,4,3,2, \mathbf{7}, \mathbf{8}, 1$ \\
\hline 4 & 0 & $\mathbf{0}, 11,8, \boldsymbol{7}, 6,5,4,3,2, \mathbf{9}, \mathbf{1 0}, 1$ \\
& 1 & $\mathbf{0}, 23,12,11,6,5,4,19,16,15,20,3,2,21,14,9,8, \mathbf{1 7}, \mathbf{1 8}, 7,10,13,22,1$ \\
\hline
\end{tabular}

Table 1: Relative orderings for trellis with signature up to 4. The intersections corresponding to the fixed point and the forcing orbits are indicated in bold type, and the intersections corresponding to the primary homoclinic orbit with code $\overline{0} 1 \overline{0}$ are indicated in italic type.

\subsection{Horseshoe homoclinic orbits with the same homoclinic braid type}

We have computed the trellis types forced by all horseshoe homoclinic orbits of signature 12 or less, and determine which pairs have the same homoclinic braid type. Table 1 presents the relative orderings of trellises of signature up to 4 , and Table 3 presents the equivalence relation for orbits of signatures up to 9 .

For orbits of signature up to 4 , the only equivalences are trivial; two homoclinic orbits have the same homoclinic braid type if and only if they have the same decoration. However the orbits of signature 5 with decorations 01 and 10, and codes $\overline{0} 1_{1}^{0} 01_{1}^{0} 1 \overline{0}$ and $\overline{0} 1_{1}^{0} 10_{1}^{0} 1 \overline{0}$ respectively, are equivalent (see Figure 5).

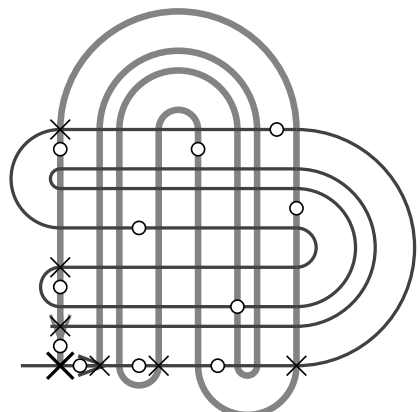

(a)

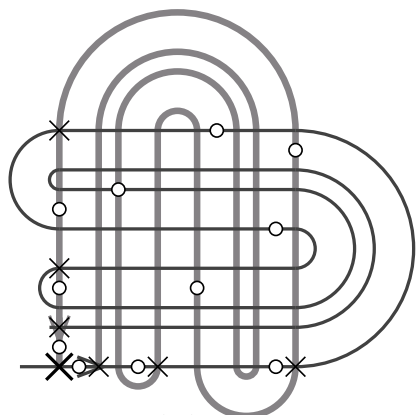

(b)

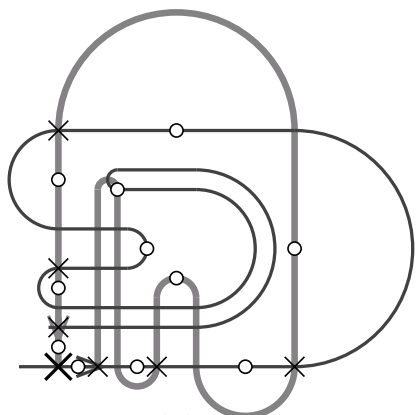

(c)

Figure 5: The horseshoe trellis and homoclinic orbits with codes (a) $\overline{0} 110111 \overline{0}$ and (b) $\overline{0} 111011 \overline{0}$, and (c) the trellis they force.

For homoclinic orbits of signature at most 7 , all orbits have the same homoclinic braid type as their time reversal. However, for orbits of signature 8, there are two pairs of orbits whose homoclinic braid type differs from that of their time reversal. This is a counterexample to the conjecture that horseshoe orbits which are time-reversals have the same braid type.

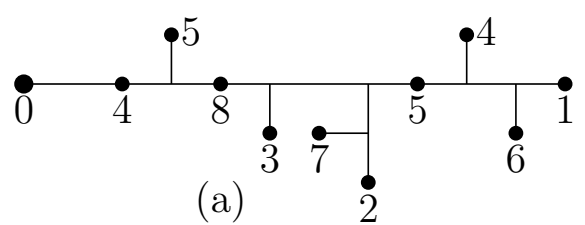

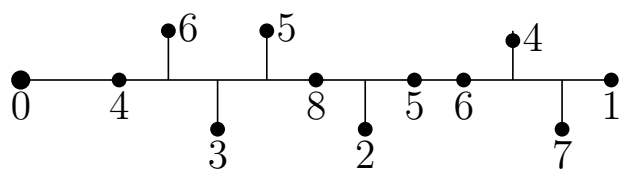

(b)

Figure 6: Topological graph representatives for the homoclinic orbits with codes (a) $\overline{0} 1_{1}^{0} 01001_{1}^{0} 1 \overline{0}$ and (b) $\overline{0} 1_{1}^{0} 10010_{1}^{0} 1 \overline{0}$.

The trellises forced by the orbits with codes $\overline{0} 1_{1}^{0} 01001_{1}^{0} 1 \overline{0}$ and $\overline{0} 1_{1}^{0} 10010_{1}^{0} 1 \overline{0}$ are not equivalent, even though these words are reverses of each other. Each trellis has 176 intersections. The graph representatives 


\begin{tabular}{|c|r|l||l|}
\hline Sig & $\# T^{V}$ & $h_{\text {top }}$ & Decorations \\
\hline 2 & 8 & 0.693147 & $*$ \\
\hline 3 & 10 & 0.528049 & $\cdot$ \\
\hline 4 & 12 & 0.434175 & 0 \\
& 24 & 0.637160 & 1 \\
\hline 5 & 14 & 0.372312 & 00 \\
& 26 & 0.585210 & 01,10 \\
& 38 & 0.589844 & 11 \\
\hline 6 & 16 & 0.327931 & 000 \\
& 28 & 0.621811 & 001,100 \\
& 39 & 0.493726 & $011,010,110$ \\
& 76 & 0.618251 & 111 \\
& 92 & 0.655290 & 101 \\
\hline 7 & 18 & 0.294274 & 0000 \\
& 29 & 0.658093 & 0001,1000 \\
& 42 & 0.450806 & $0011,0010,0100,1100$ \\
& 58 & 0.471989 & 0110 \\
& 70 & 0.549761 & $0111,0101,1110,1010$ \\
& 122 & 0.591665 & 1101,1011 \\
& 134 & 0.604018 & 1111 \\
& 218 & 0.684471 & 1001 \\
\hline
\end{tabular}

Table 2(a): Equivalences for homoclinic orbits with signature up to 7 .

\begin{tabular}{|c|r|l|ll|}
\hline Sig & $\# T^{V}$ & $h_{\text {top }}$ & Decorations & \\
\hline 8 & 19 & 0.267727 & 00000 & \\
& 31 & 0.676320 & 00001,10000 & \\
& 43 & 0.458765 & $00011,00010,01000,11000$ \\
& 60 & 0.410427 & $00110,00100,01100$ \\
& 72 & 0.563961 & $00111,00101,11100,10100$ \\
100 & 0.535252 & $01101,01110,01010,10110$ \\
& 112 & 0.518203 & $01111,01011,11010,11110$ \\
& 176 & 0.612495 & 01001 \\
& 252 & 0.646515 & 11001 \\
& 176 & 0.612495 & 10010 \\
& 252 & 0.646515 & 10011 \\
& 208 & 0.576220 & 11011 \\
& 252 & 0.612102 & 11111 \\
& 268 & 0.625290 & 11101,10111 \\
& 336 & 0.650772 & 10101 \\
& 472 & 0.690998 & 10001 \\
\hline
\end{tabular}

Table 3(b): Equivalences for homoclinic orbits with signature 8. 


\begin{tabular}{|c|c|c|c|}
\hline Sig & $\# T^{V}$ & $h_{\text {top }}$ & Decorations \\
\hline \multirow[t]{27}{*}{9} & 22 & 0.246163 & 000000 \\
\hline & 34 & 0.684979 & 000001, 100000 \\
\hline & 46 & 0.485379 & 000011, 000010, 010000, 110000 \\
\hline & 62 & 0.376307 & 000110, 000100, 001000, 011000 \\
\hline & 74 & 0.597015 & 000111, 000101, 111000, 101000 \\
\hline & 82 & 0.398273 & 001100 \\
\hline & 94 & 0.537060 & 001101, 001110, 001010, 001001, 011100, 010100, 101100, 100100 \\
\hline & 106 & 0.504368 & 001111, 001011, 110100, 111100 \\
\hline & 150 & 0.604226 & 011001, 100110 \\
\hline & 162 & 0.484357 & 011011, 011010, 011110, 010110, 110110 \\
\hline & 198 & 0.537481 & 011111, 010111, 111110, 111010 \\
\hline & 214 & 0.563076 & 011101, 010101, 101010, 101110 \\
\hline & 182 & 0.502700 & 010010, 010011, 110010 \\
\hline & 430 & 0.659895 & 010001 \\
\hline & 510 & 0.671444 & 110001 \\
\hline & 310 & 0.548079 & 110011 \\
\hline & 378 & 0.583547 & 110111, 110101, 111011, 101011 \\
\hline & 446 & 0.603536 & 111101, 101111 \\
\hline & 458 & 0.607837 & 111111 \\
\hline & 650 & 0.666417 & 111001 \\
\hline & 722 & 0.668611 & 101001 \\
\hline & 725 & 0.670332 & 101101 \\
\hline & 722 & 0.668611 & 100101 \\
\hline & 650 & 0.666417 & 100111 \\
\hline & 430 & 0.659895 & 100010 \\
\hline & 510 & 0.671444 & 100011 \\
\hline & 982 & 0.692607 & 100001 \\
\hline
\end{tabular}

Table 3(c): Equivalences for homoclinic orbits with signature 9 . 
are shown in Figure 6. The topological entropy of both these orbits is $\log \lambda$, where $\lambda$ is the largest root of the polynomial

$$
\lambda^{13}-2 \lambda^{12}+2 \lambda^{8}+\lambda^{7}-4 \lambda^{5}-2 \lambda^{4}+2 \lambda^{2}+2 \lambda-2 .
$$

Numerically, $\lambda_{\max } \approx 1.845$, giving $h_{\text {top }}>0.612$.

Another counterexample is given by the orbits with codes $\overline{0} 1_{1}^{0} 11001{ }_{1}^{0} 1 \overline{0}$ and $\overline{0} 1{ }_{1}^{0} 10011_{1}^{0} 1 \overline{0}$. These trellises each have 252 intersections. The topological entropy of both these orbits is $\log \lambda$, where $\lambda$ is the largest root of the polynomial

$$
\lambda^{13}-2 \lambda^{12}+3 \lambda^{7}-4 \lambda^{6}+4 \lambda^{5}+2 \lambda^{4}+2 \lambda-2 .
$$

Numerically, $\lambda_{\max } \approx 1.909$, giving $h_{\mathrm{top}}>0.646$.

\subsection{The forcing relation}

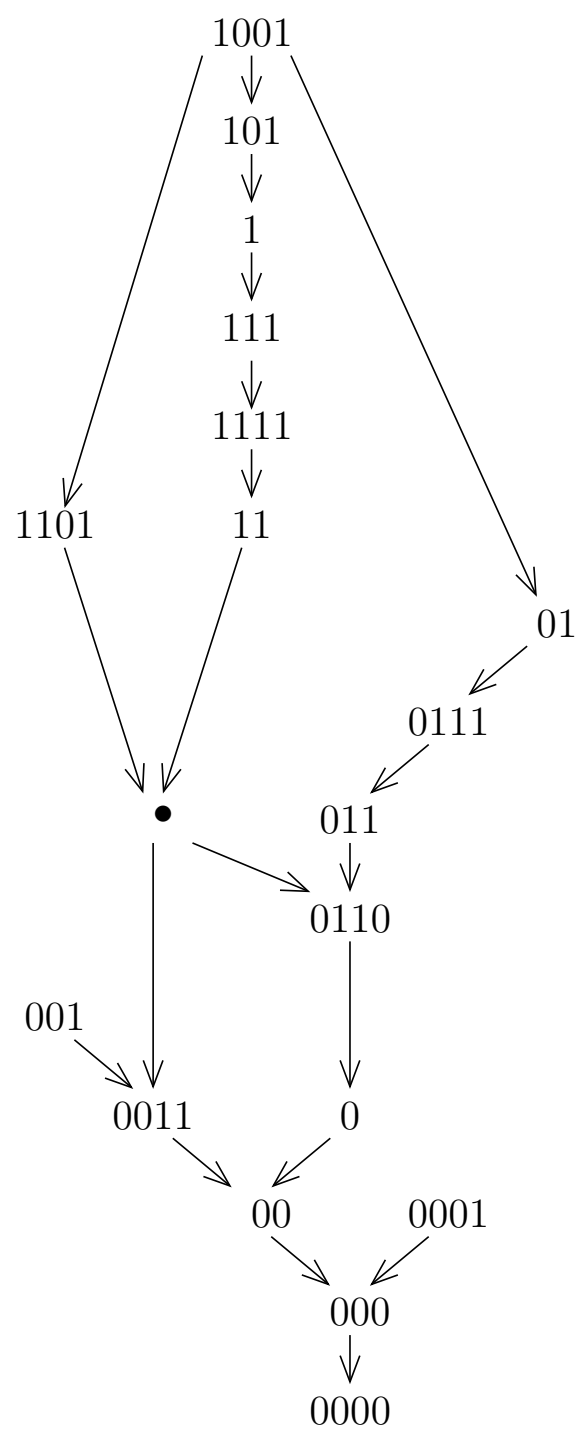

Figure 7: Decorations and scopes of homoclinic braid types of signature up to 7 and the forcing relation between them.

Figure 7 shows the forcing relation between horseshoe homoclinic orbits of signature 7 or less. Each homoclinic orbit is specified by its decoration, and the scope of the decoration is also given. Only one 
decoration is given for each equivalence class of homoclinic braid types (so, for example, the decoration 10 is not included, since it is equivalent to 01 ).

We give two examples illustrating the computation of Figure 7 the interpretation of the results.

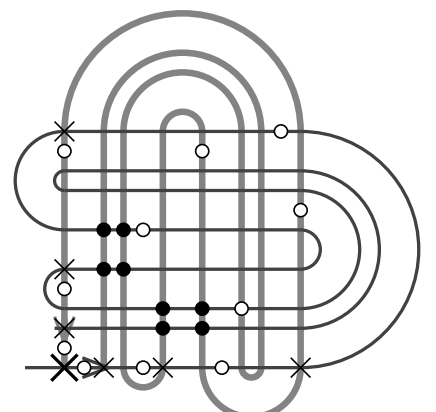

(a)

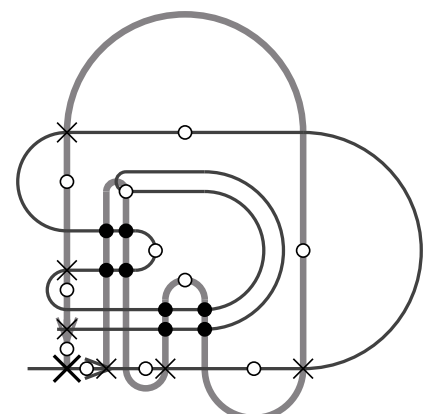

(b)

Figure 8: The homoclinic orbit $\overline{0} 110111 \overline{0}$ forces the orbits $\overline{0} 1_{1}^{0} 0_{1}^{0} 1 \overline{0}$.

Example 5.1 Figure 8 shows the horseshoe trellis, on which the orbit $H$ with code $\overline{0} 110111 \overline{0}$ (decoration 01 ) has been marked with white dots, and the intersections on the orbits with codes $\overline{0} 1_{1}^{0} 0_{1}^{0} 1 \overline{0}$ with black dots. On performing a pruning isotopy to obtain the trellis forced by the orbit $P$, it can be seen that the marked intersections persist. Therefore the homoclinic braid type with decoration 01 forces the homoclinic braid type with decoration 0 . From Figure 8 it can also be seen that the only other forced homoclinic braid type with signature less than 5 is that with decoration 00 .

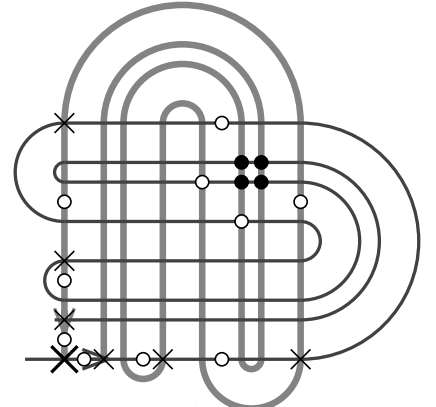

(a)

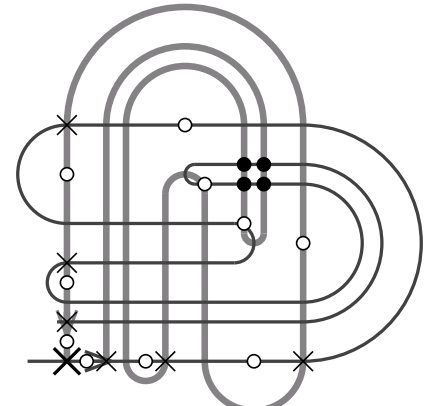

(b)

Figure 9: The homoclinic orbit $\overline{0} 11111 \overline{0}$ forces the orbits $\overline{0} 1_{1}^{0} 11_{1}^{0} 1 \overline{0}$.

Example 5.2 It is possible to compute braid types of arbitrary signature forced by any homoclinic orbit. In Figure 9, we show the trellis of signature 5 forced by the homoclinic orbit $\overline{0} 11111 \overline{0}$ which has decoration 1 and signature 4, allowing us to compute the braid types of signature up to 5 forced by the orbit. In particular, we see that the orbit $\overline{0} 11111 \overline{0}$ forces the orbits $\overline{0} 1_{1}^{0} 11_{1}^{0} 1 \overline{0}$, so the homoclinic braid type with decoration 1 forces the homoclinic braid type with decoration 11. The other homoclinic braid types with signature less than 5 forced by the braid type with decoration 1 have decorations $\cdot, 0$ and 00 .

\section{Conclusions and further research}

We have described a numerical method for determining the forcing relation for homoclinic orbits of the Smale horseshoe map using trellises. The method is exact, in that is considers orbits given in terms of their symbolic codes, and The numerical techniques can also be used to determine forcing relations between homoclinic and heteroclinic orbits of general surface homeomorphisms. 
One of the motivations for this work was to help in determining the forcing relation between periodic orbits of the Smale horseshoe map, and between homoclinic and periodic orbits. Unfortunately, while it is fairly straightforward to compute a train track for a pseudo-Anosov periodic orbit, it is less straightforward to determine the codes of the periodic orbits forced by a given homoclinic orbit. The main difficulties is that there may be many periodic orbits of the Smale horseshoe map with the same braid type, and that pruning the trellis destroys the partition of the regions $R_{0}$ and $R_{1}$, making it difficult to recover the braid type from a trellis or its graph representative.

There are also number of important related computational problems for which no methods are currently available. On the combinatorial side, it is also important to develop methods for computing the topological graph representative of a trellis type; this has been implemented for horseshoe trellises, but not for trellises on general surfaces. It is also important to have an automatic procedure for generating a combinatorial trellis from numerically-computed stable and unstable manifolds. Unfortunately, naïve methods fail since a numerically computed trellis may have orbits not present in the actual system, and may even be inconsistent. An extension of the trellis package is planned which will allow the dynamics forced by a numerically computed trellis to be found.

\section{References}

[1] Viviane Baladi and David Ruelle. An extension of the theorem of milnor and thurston on the zeta functions of interval maps. Ergodic Theory Dynamical Systems, 14(4):621-632, 1994.

[2] Mladen Bestvina and Michael Handel. Train-tracks for surface homeomorphisms. Topology, 34(1):109-140, 1995.

[3] Philip Boyland. Rotation sets and monotone periodic orbits for annulus homeomorphisms. Comment. Math. Helv., 67(2):203-213, 1992.

[4] Philip L. Boyland. Isotopy stability of dynamics on surfaces. In Marcy Barge and Krystyna Kuperberg, editors, Geometry and Topology in Dynamics, number 246 in Contemporary Mathmatics, pages 17-45, Providence, Rhode Island, 1999. American Mathematical Society.

[5] Pieter Collins. Entropy-minimising models of surface diffeomorphisms relative to homoclinic and heteroclinic orbits. To appear in Dynam. Sys.

[6] Pieter Collins. Software package "trellis". Available from http://www.cwi.nl/ collins/.

[7] Pieter Collins. Symbolic dynamics from homoclinic tangles. Intern. J. Bifur. Chaos, 12(3):605-617, 2002.

[8] Pieter Collins. Dynamics of surface diffeomorphisms relative to homoclinic and heteroclinic orbits. Dynam. Sys., 19(1):1-39, 2004.

[9] André de Carvalho. Pruning fronts and the formation of horseshoes. Ergodic Theory Dynamical Systems, 19(4):851-894, 1999.

[10] André de Carvalho and Toby Hall. The forcing relation for horseshoe braid types. Experimental Math., 11(2):271-288, 2002.

[11] André de Carvalho and Toby Hall. Braid forcing and star-shaped train tracks. Topology, 43(2):247287, 2004.

[12] Robert Devaney and Zbigniew Nitecki. Shift automorphisms in the Hénon mapping. Comm. Math. Phys., 67(2):137-146, 1979.

[13] John Franks and Michael Misiurewicz. Cycles for disk homeomorphisms and thick trees. In Nielsen Theory and Dynamical Systems, number 152 in Contemporary Mathematics, pages 69-139, 1993.

[14] Michael Handel. Global shadowing of pseudo-Anosov homeomorphisms. Ergodic Theory Dynamical Systems, 5(3):373-377, 1985.

[15] Michael Handel. A fixed-point theorem for planar homeomorphisms. Topology, 38(2):235-264, 1999. 
[16] Helen Hulme. Finite and infinite braids: a dynamical systems approach. $\mathrm{PhD}$ thesis, University of Liverpool, 2000.

[17] Anatole Katok. Lyapunov exponents, entropy and periodic orbits for diffeomorphisms. Inst. Hautes Études Sci. Publ. Math., 51:137-173, 1980.

[18] Eiko Kin. The third power of the smale horseshoe induces all link types. J. Knot Theory Ramifications, 9(7):939-953, 2000.

[19] Jérôme Los. Pseudo-Anosov maps and invariant train tracks in the disc: A finite algorithm. Proc. London Math. Soc. (3), 66(2):400-430, 1993.

[20] F. Allan McRobie and J. M. T. Thompson. Knot-types and bifurcations sequences of homoclinic and transient orbits of a single-degree-of-freedom driven oscillator. Dynam. Stab. Sys., 9:223-251, 1994.

[21] John Milnor and William Thurston. On iterated maps of the interval. In Dynamical systems, number 1342 in Lecture Notes in Math., pages 465-563, Berlin, 1988. Springer.

[22] Vered Rom-Kedar. Homoclinic tangles - classification and applications. Nonlinearity, 7(2):441-473, 1994.

[23] Alexander N. Sharkovskiı. Coexistence of cycles of a continuous map of the line into itself. Ukrain. Mat. Zh., 16(1):61-71, 1964. Russian.

[24] William P. Thurston. On the geometry and dynamics of diffeomorphisms of surfaces. Bull. Amer. Math. Soc. (N.S.), 19(2):417-431, 1988. 